\title{
BAJO LA INFLUENCIA DEL BUDISMO Y EL ANIMISMO: CUERPO, ENFERMEDAD, MEDICINA Y CUIDADOS EN EL CINE DE APICHATPONG WEERASETHAKUL (LOS EJEMPLOS DE SYNDROMES AND A CENTURY, 2006 Y CEMETERY OF SPLENDOUR, 2015)
}

\section{Under the influence of Buddhism and animism: body, disease, medicine and care in the cinema of Apichatpong Weerasethakul (the examples of Syndromes and a Century, 2006 and Cemetery of Splendour, 2015)}

Álvaro MALAINA

Departamento de Sociología: Metodología y Teoría. Facultad de C. Políticas y Sociología, Universidad Complutense de Madrid (España).

Autor para correspondencia: Álvaro Malaina

Correo electrónico: alvaromm@pdi.ucm.es

Recibido: 13 de mayo de 2020

Aceptado: 16 de junio de 2020

\section{Resumen}

El modelo biomédico occidental ha sido ampliamente retratado en el cine y las series televisivas. El artículo busca poner ejemplos de modelos terapéuticos alternativos al biomédico que aún tienen mucha presencia en culturas no occidentales, combinándose a menudo con el modelo biomédico y que no han sido tan reflejados en el cine. Ponemos ejemplos de dos películas del director tailandés Apichatpong Weerasethakul, Syndromes and a Century (Sang Sattawat, 2006) y Cemetery of Splendor (Rak Ti Khon Kaen, 2015). Estas películas nos muestran cómo en la región tailandesa de Isan se combina el modelo biomédico, de origen occidental pero ya plenamente asentado en el país, con técnicas meditativas y prácticas mágicas influidas por el budismo theravada y el animismo de origen laosiano practicados en la región.

Palabras clave: cine; medicina; budismo; animismo 
BAJO LA INFLUENCIA DEL BUDISMO Y EL ANIMISMO: CUERPO, ENFERMEDAD, MEDICINA Y CUIDADOS EN EL CINE DE APICHATPONG WEERASETHAKUL

ÁLVARO MALAINA

\section{Summary}

The Western biomedical model has been widely portrayed in film and television series. The article seeks to give examples of alternative therapeutic models to biomedical that still have a strong presence in non-western cultures, often combining with the biomedical model and that have not been so reflected in the cinema. We give examples of two films by the Thai director Apichatpong Weerasethakul, Syndromes and a Century (Sang Sattawat, 2006) and Cemetery of Splendour (Rak Ti Khon Kaen, 2015). These films show us how in the Thai region of Isan the biomedical model of Western origin but already fully established in the country is combined with meditative techniques and magical practices influenced by Theravada Buddhism and animism of Laotian origin practiced in the region.

Keywords: cinema; medicine; buddhism; animism.

Introducción: la medicina en el cine de Apichatpong Weerasethakul

La medicina y las prácticas hospitalarias que siguen el modelo biomédico dominante en los países occidentales tienen un lugar propio en el cine y las series televisivas de Europa y Estados Unidos, hasta configurar un propio subgénero cinematográfico que goza de mucha popularidad. Nuestro artículo busca reflejar modelos alternativos al biomédico en cine de países no occidentales. Al mismo tiempo buscamos reflejar cómo estos modelos y prácticas terapéuticas nacidas de otras culturas a menudo se intersectan, combinan y ensamblan con el modelo biomédico, de raigambre occidental pero que ya es plenamente global. Esta medicina alternativa tiene poco reflejo en el cine euroamericano o cuando lo hace se inscribe o bien en un género fantástico o como documental «exótico» proselitista. Pocas veces refleja este modelo terapéutico no occidental en su propia naturalidad y especialmente en su entrecruzamiento con la medicina que podemos denominar dominante y ortodoxa, sin tomar partido en su favor o en su contra. Ponemos el ejemplo del cine del director tailandés Apichatpong Weerasethakul (Bangkok, 1970), que recientemente ha conocido mucho éxito entre la crítica occidental y que ganara en 2010 la Palma de Oro del Festival de Cannes con su película Tío Boonmee recuerda sus vidas pasadas (Lung Bunmi Raluek Chat, 2010).
La medicina y los cuidados del cuerpo tienen un papel central en el cine del director tailandés, que se caracteriza por un estilo muy personal que combina un hiperrealismo casi documental con elementos oníricos y fantásticos ${ }^{1}$. La explicación de su interés por la medicina está en su propia biografía como hijo de médicos y sus memorias de infancia, criado en entornos de hospital. Apichatpong ha sabido combinar en muchas de esas películas estas experiencias y memorias de una medicina de corte occidental como la practicada por sus padres, con toda una serie de prácticas médicas y terapéuticas de la cultura local de su región de origen, Isan. La región tailandesa del nordeste, Isan, es una de las más pobres del país y su cultura se nutre en gran parte por el budismo theravada, religión oficial del país, pero también por el animismo y chamanismo de origen khmer practicado en el vecino Laos con quien tiene muchos vínculos culturales e históricos ${ }^{2,3}$. Apichatpong se aproxima a las prácticas terapéuticas alternativas de Isan desde una visión neutra, laica, escéptica y distanciada, no desde el proselitismo. Las aborda como un observador para quien gran parte de las creencias locales como la de la reencarnación $\mathrm{u}$ otras no tienen lógica, pero que constata sin embargo que impregnan a buena parte de las gentes de su lugar natal, formando parte de su paisaje antropo-sociológico y desea reflejarlo en su cine artísticamente. Y esa es la aportación desde el prisma médico de su obra: nos ofrece visiones 
BAJO LA INFLUENCIA DEL BUDISMO Y EL ANIMISMO: CUERPO, ENFERMEDAD, MEDICINA Y CUIDADOS EN EL CINE DE APICHATPONG WEERASETHAKUL

ÁLVARO MALAINA

del cuerpo y la enfermedad, de los hospitales y los cuidados, combinando, sin jerarquizar, en un mismo plano horizontal, acercamientos propios del modelo biomédico occidental más racionalizado con acercamientos propios de influencias budistas y animistas con visiones mágicas, míticas y místicas de la salud y la enfermedad. Sin un rechazo cientificista eurocéntrico de éstas últimas pero sin proselitismo "orientalista» ${ }^{4}$ naif tampoco. Este ensamblaje no vertical convierte sus películas en artefactos complejos o «rizomas» cinematográficos de profundidad etnográfica ${ }^{5}$, como hemos descrito siguiendo al filósofo francés Deleuze ${ }^{6}$. El cine de Apichatpong puede generar así cierta confusión en el espectador, particularmente el occidental, pero nos aporta en cambio mucha información antropológica y sociológica de las realidades culturales no occidentales en la era de la globalización y de sus "ensamblajes globales» ${ }^{7}$ que combinan formas y normas globales de racionalidad en entornos culturales situados.

Vamos a analizar la presencia de la medicina especialmente en dos de sus largometrajes: Syndromes and a Century (Sang Sattawat, 2006) y Cemetery of Splendour (Rak Ti Khon Kaen, 2015).

\section{Fichas técnicas}

Título: Syndromes and a Century.

Título original: Sang Sattawat.

País: Tailandia.

Año: 2006.

Director: Apichatpong Weerasethakul.

Música: Kantee Anantagant.

Fotografía: Sayombhu Mukdeeprom.

Montaje: Lee Chatametikool.

Guión: Apichatpong Weerasethakul.

Intérpretes: Arkanae Cherkam, Jaruchai lamaram, Sakda Kaewbuadee, Nu Nimsomboon, Jenjira Pongpas.

Color: color.

Duración: 105 minutos.

Género: drama; romance, medicina, historias cruzadas.
Idioma original: Tailandés.

Sinopsis: Inspirada en la historia de amor real de los padres del director, ambos médicos, y los recuerdos del propio director. La primera parte se desarrolla en un hospital rural y la segunda en un hospital de Bangkok, mostrando escenas cotidianas de amor y trabajo de ambos médicos en dichos hospitales.

Productoras: Anna Sanders Films, Backup Media (in association with), Centre National du Cinéma et de l'Image Animée. Fonds Sud Cinéma, French Ministry of Foreign Affairs, Illuminations Films, Kick the Machine, Ministère de la Culture et de la Communication, New Crowned Hope, TIFA.

Enlaces: https://www.filmaffinity.com/es/ film618062.html

https://www.imdb.com/title/tt0477731

\section{Trailer}

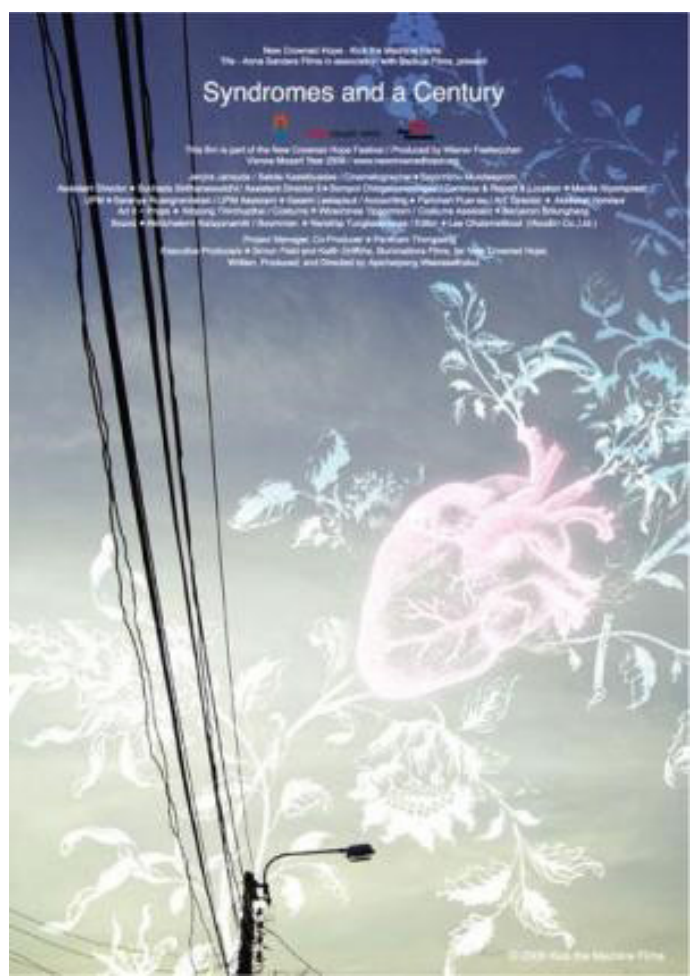


BAJO LA INFLUENCIA DEL BUDISMO Y EL ANIMISMO: CUERPO, ENFERMEDAD, MEDICINA Y CUIDADOS EN EL CINE DE APICHATPONG WEERASETHAKUL

ÁLVARO MALAINA

Título: Cemetery of Splendour.

Título original: Rak Ti Khon Kaen.

País: Tailandia.

Año: 2015.

Director: Apichatpong Weerasethakul.

Música: Apichatpong Weerasethakul.

Fotografía: Diego García.

Montaje: Lee Chatametikool.

Guion: Apichatpong Weerasethakul.

Intérpretes: Jenjira Pongpas, Banlop Lomnoi, Jarinpattra Rueangram, Petcharat Chaiburi, Tawatchai Buawat.

Color: Color.

Duración: 122 minutos.

Género: drama; fantástico; surrealismo; medicina.

Idioma original: tailandés.

Sinopsis: una solitaria ama de casa de mediana edad atiende a un soldado con la enfermedad del sueño, cayendo en alucinaciones que desencadenan sueños extraños, fantasmas y romances.

Productoras: Kick the Machine, Anna Sanders Films (co-production), Match Factory Productions (co-production), Geißendörfer Film- und Fernsehproduktion (GFF) (co-production), ZDF/ Arte (co-production), Astro Shaw (co-production), Asia Culture Centre-Asian Arts Theatre (co-production), Detalle Films (co-production), Louverture Films (co-production), Tordenfilm AS (co-production), Centre National du Cinéma et de I'Image Animée (support), Illuminations Films, The Match Factory.

Enlaces: https://www.filmaffinity.com/es/ film435424.html https://www.imdb.com/title/tt2818654

\section{Trailer}

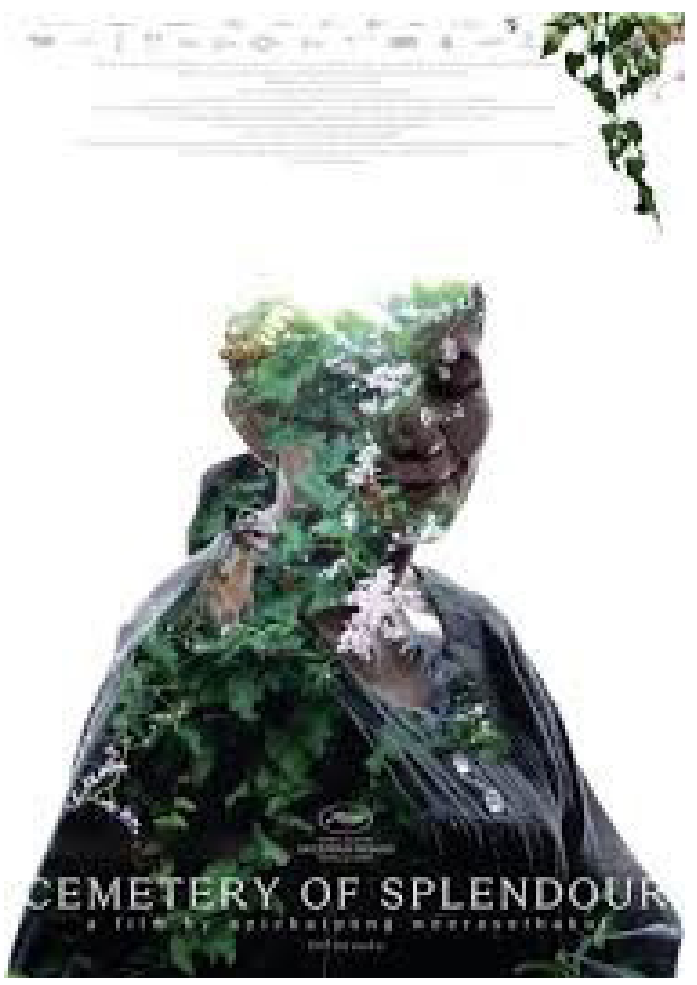

Nuestro marco teórico: el ensamblaje complejo del modelo biomédico con el modelo terapéutico animista y budista de la región tailandesa de Isan

El modelo biomédico dominante en los países occidentales en su esencia reduce la enfermedad al cuerpo físico del paciente, entendida como alteración o desviación del correcto funcionamiento de sus órganos. Este modelo debido a su eficacia terapéutica se ha extendido por todo el mundo, incluyendo a los países no occidentales. Sin embargo, en algunos de estos países, sobre todo de Asia, coexiste en ocasiones o se combina con un modelo terapéutico alternativo, influido por el hinduismo y el budismo, así como por religiones y creencias locales de tipo animista o chamanista. Este modelo se centraría en una visión holística de la salud que considera que además del cuerpo físico y su componente orgánico (o físico-psíquico 
BAJO LA INFLUENCIA DEL BUDISMO Y EL ANIMISMO: CUERPO, ENFERMEDAD, MEDICINA Y CUIDADOS EN EL CINE DE APICHATPONG WEERASETHAKUL

ÁLVARO MALAINA

o incluso físico-psíquico-social como en variantes más recientes del modelo biomédico occidental, si se introducen factores psicológicos y sociológicos como desencadenantes o agravantes de enfermedades) habría un cuerpo espiritual o energético, donde puede radicar la enfermedad y el origen del sufrimiento de la persona, si se alteran sus equilibrios esenciales. Este paradigma médico del espíritu o la energía que se encuentra desde tiempo inmemorial en muchos países de Asia rige desde sistemas terapéuticos sistemáticos y tradicionales consolidados como el ayurveda indio (con su concepto de doshas o humores vitales) hasta la medicina tradicional china (con su concepto del chi o energía vital), incluyendo también toda una serie de prácticas populares mágicas basadas en supersticiones locales y centradas en uso de amuletos, ungüentos $u$ ofrendas en el templo a fin de obtener salud y buenos augurios.

En el caso concreto de la región nororiental de Tailandia, Isan, donde creció Weerasethakul, las concepciones alternativas de la salud, la enfermedad y la curación se ven influidas por un sincretismo ecléctico que combina creencias de origen hinduista en la reencarnación, prácticas de meditación budista propias del budismo theravada y toda una serie de leyendas, mitos y prácticas mágicas tradicionales provenientes del animismo chamanista de origen Khmer que por proximidad con Laos ha tenido influencia histórica en la región.

Los conceptos de "karma» y reencarnación de origen hinduista y también presentes en gran parte en el budismo en cuanto deriva herética del hinduismo, son también centrales a la concepción de la enfermedad en la región de Isan. Según la medicina budista e hinduista por ejemplo, la enfermedad o malestar del paciente pueden ser causadas por un karma negativo, que es una ley cósmica de causalidad según la cual las acciones negativas en la vida pasada o en otras vidas anteriores del individuo tienen efectos negativos en la vida presente del mismo. Esta concepción de la salud basada en la idea del karma tiene una raigambre muy importante en las prácticas terapéuticas populares de millones de personas por lo que su valor antropológico es indudable. Es muy interesante por tanto ver cómo el cine lo refleja, insistiendo siempre en no caer en orientalismos vanos y sin entrar a juzgar la pertinencia o no de estas terapias alternativas, terreno que aquí no nos ocupa, sino para resaltar su valor cultural y antropológico ligados a pueblos no occidentales. Este tipo de causalidad cósmica está presente en toda la filmografía del director tailandés y especialmente es clave en su película Tío Boonmee recuerda sus vidas pasadas, cuando el protagonista Boonmee, moribundo, dice que cree que la culpa de su enfermedad renal es que «mató a demasiados comunistas» durante el conflicto entre las fuerzas armadas tailandesas y las milicias comunistas próximas a Laos en la región de Isan que produjo miles de muertos.

El budismo incide también en el sufrimiento como una de las nobles verdades formuladas por Buda en su enseñanza originaria ${ }^{8}$. La primera noble verdad es dukkha, el dolor o sufrimiento como inherentes a la existencia. Pero la segunda noble verdad es la verdad de la causa samudaya del sufrimiento que es el deseo y el apego a la existencia misma, o incluso a la no-existencia. La tercera noble verdad es la verdad de la cesación y extinción, nirodha, del dolor y el sufrimiento mediante la cesación y extinción del deseo. La cuarta verdad es la de la existencia de una vía, mārga, para la extinción de dukkha, conocida como Óctuple Sendero, basado en una conducta ética, un recogimiento meditativo y un conocimiento superior. Consiste en ocho prácticas o caminos simultáneos que constituirían la «terapia» budista propia contra el sufrimiento y el dolor. Una de las prácticas consiste en una serie de técnicas meditativas de atención y concentración perfectas para la extinción del deseo y del apego a sentimientos, pensamientos, acciones ${ }^{9}$. Una de las más practicadas por la escuela budista Theravada, predominante en países del Sudeste de Asia, es

Rev. Med. Cine. 2021; 17 (2), 123-132 Ediciones Universidad de Salamanca / అ®@ J. Med. Mov., 2021; 17 (2), 123-132 
BAJO LA INFLUENCIA DEL BUDISMO Y EL ANIMISMO: CUERPO, ENFERMEDAD, MEDICINA Y CUIDADOS EN EL CINE DE APICHATPONG WEERASETHAKUL

ÁLVARO MALAINA

la meditación vipassana que busca superar el sufrimiento psicosomático mediante una autoexploración consciente de los procesos mentales y corporales cambiantes a fin de auto-purificarse tomando conciencia de su impermanencia (anitya) y de la inexistencia del sí mismo (anatman). En muchas de las películas de Apichatpong, como en Cemetery of Splendour, se nos muestran sesiones de meditación como vía de sanación.

Por su parte, el animismo chamánico de la región de Isan que retrata a menudo Weerasethakul también comparte este paradigma de la dimensión espiritual del ser humano y la posibilidad de una «curación espiritual» de las dolencias. En las creencias denominadas «animistas», todo objeto o fenómeno natural estaría dotado de alma y así el ser humano tendría dos cuerpos, el biofísico que se mueve en el mundo denso de la vida cotidiana, y un cuerpo astral o espíritu o alma que se mueve el mundo sutil de los sueños o que se libera por ejemplo tras rituales chamánicos como la ingesta de plantas psicodélicas ${ }^{10}$. Habría así un mundo natural y un mundo sobrenatural interconectados, donde los espíritus de vivos, muertos, plantas, objetos o animales, influyen en el mundo material produciendo enfermedades y sufrimiento si son malignos, o éxtasis y curaciones si son bondadosos. El chamán es el sanador capaz de viajar mediante técnicas de trance (que van desde el son de tambores hasta la ingesta de plantas psicotrópicas) al mundo paralelo de los espíritus, a fin de comunicar con ellos, arreglar conflictos y solucionar males y enfermedades del mundo material. En la segunda parte de la película de Apichatpong Tropical Malady (Sud Pralad, 2004) se nos muestra por ejemplo cómo el espíritu de un chamán es capaz de encarnarse en el cuerpo de un tigre que perturba a los habitantes de un poblado.

La medicina que nos muestra Apichatpong en su cine tiene una fuerte raigambre en el modelo biomédico occidental, pero lo conecta con las concepciones animistas y budistas de su región natal, creando ensamblajes complejos, donde una no se reduce ni reduce a la otra, sino que ambas se mantienen independientes y se retroalimentan en su heterogeneidad. Vamos a poner ejemplos de dos sus películas, Syndromes and a Century y Cemetery of Splendour.

\section{Syndromes and a Century: memorias de medicina ortodoxa y alternativa}

Syndromes and a Century es una película en la que Apichatpong recuerda su propia infancia en Kohn Kaen, en los ambientes hospitalarios donde trabajaban sus padres. La película está dividida en dos partes. La primera parte se desarrolla en un hospital rural en mitad de la jungla. Las prácticas biomédicas se incorporan, pero todavía tienen mucho peso las terapias alternativas de la población local, sobre todo de los monjes budistas theravada. En la segunda parte se nos muestra un hospital urbano, en un entorno ya desarrollado y moderno. Apichatpong nos muestra que, a pesar de la modernidad del hospital, siguen existiendo incluso entre el colectivo médico profesional prácticas terapéuticas alternativas propias de cosmovisiones budista y animista.

Pongamos varios ejemplos. En una escena (Foto 1), una consulta en el hospital rural con la naturaleza esplendorosa tras la ventana, un monje budista anciano en túnica azafrán le cuenta a la doctora cómo cree que la culpa de que se cayera de la cama la otra noche y de que le duelan las articulaciones es de los espíritus de los pollos, a los que maltrataba cuando era niño. Esos espíritus le visitan en sueños, explica, le poseen y le maldicen. Le persiguen buscando venganza y él intenta escapar. En su sueño, sigue explicando el monje, le crecieron alas y él mismo se convirtió en pollo y fueron los pollos los que le despertaron, le empujaron y tiraron de la cama. Observamos aquí esa mezcla de budismo y animismo/chamanismo que estará presente de forma mucho más explícita en películas posteriores del director tailandés y que es una constante de su filmografía. Los elementos racionales del modelo biomédico son incorporados al final en palabras de la doctora, 
BAJO LA INFLUENCIA DEL BUDISMO Y EL ANIMISMO: CUERPO, ENFERMEDAD, MEDICINA Y CUIDADOS EN EL CINE DE APICHATPONG WEERASETHAKUL

ÁLVARO MALAINA

que tras escuchar el relato del monje con atención le pregunta: «¿Aún te duelen las articulaciones? ¿Comes mucho pollo?».

El anciano monje dice que aún le duele y que come más pollo del que debería para reducir su mal karma. El médico le da entonces la explicación científica y racional: hay gran cantidad de ácido úrico en las aves de corral, se acumula en las articulaciones si se come demasiado. Le pide entonces hacer pruebas de sangre para confirmarlo. El monje acepta pero en una réplica inesperada saca de una bolsa unas raíces que entrega a la doctora para que prepare una pócima porque siente que ella tiene problemas emocionales y de dinero (una escena de la película nos había mostrado precisamente poco antes una conversación de la doctora fuera del hospital con un hombre sobre un dinero prestado que no le había devuelto). La doctora las acepta y en un nuevo requiebro de lo esperado, el anciano monje le pide a su vez a la doctora que le recete pastillas para dormir y vitaminas para él y para los monjes de su templo, a lo que la doctora se niega porque toda prescripción debe ser individualizada.

Vemos aquí ya la sorpresa y extrañeza que deriva del ensamblaje no trivial de elementos heterogéneos e incluso contradictorios, ciencia y mito, monjes con sueños extraños que creen ser poseídos por espíritus de aves y a la vez piden medicación sin miramientos y aún en contra de la legislación.

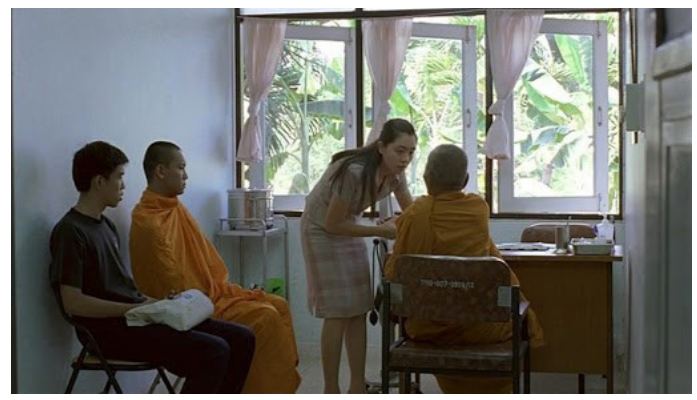

Foto 1. Consulta médica en el hospital rural con un paciente monje
En todo momento en la película aparecen destellos de la religión y superstición local entremezclados con elementos de racionalidad y modernidad. En una escena (Foto 2) de la segunda parte en el hospital moderno de ciudad, uno de los médicos, una doctora que bebe alcohol escondido en una prótesis porque esa noche va a salir en un programa televisivo, no duda en ensayar una curación «chakra» para un joven afectado de una intoxicación y que aún no se ha recuperado a pesar de varios meses de tratamiento biomédico estándar, una curación que busca canalizar la energía del sol transfiriéndosela a través del chakra de la cabeza. Esta terapia alternativa incluye técnicas de visualización, se invita al joven a imaginar que camina por un bosque, encuentra una cascada y sumerge sus manos en el agua, sintiendo cómo el agua purifica y limpia su mente, antes de vaporizarse en el cielo. Durante esta terapia otra de las doctoras mira fijamente a la cámara en silencio, volviendo la escena aún más enigmática, a pesar de desarrollarse en el aparentemente aséptico y racionalizado hospital urbano. En más vueltas de tuerca misteriosas, la doctora le pregunta si se encuentra mejor y el joven dice que no siente nada. La doctora le ofrece un vaso de alcohol pero el joven abandona la sala. El médico que le llevaba tratando dos meses confiesa a la doctora que también había ensayado con él la terapia de la cascada sin éxito.

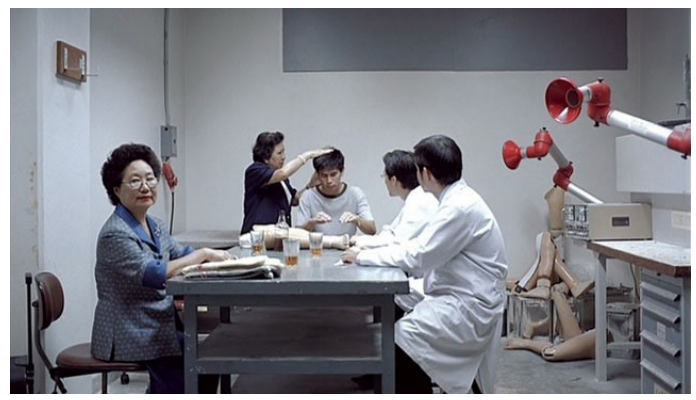

Foto 2. Terapia energética chakra practicada por una doctora en el hospital urbano 
BAJO LA INFLUENCIA DEL BUDISMO Y EL ANIMISMO: CUERPO, ENFERMEDAD, MEDICINA Y CUIDADOS EN EL CINE DE APICHATPONG WEERASETHAKUL

ÁLVARO MALAINA

El joven se dedica después a jugar al tenis en el pasillo del hospital. El médico Nohng, uno de los protagonistas de la película, le pregunta qué va a hacer en su vida. Dice que por su problema no puede llevar una vida normal, quizá en la próxima vida, le responde el joven. La escena introduce así también el tema de la reencarnación, presente en muchas escenas de la película, en las conversaciones mismas entre doctores y pacientes, insertándose con gran naturalidad en el ensamblaje complejo que aporta la película conectando distintas cosmovisiones de la salud, la enfermedad y las terapias, siempre captadas desde el prisma de la personalidad artística del cineasta y sin pretensión de objetividad.

\section{Cemetery of Splendour: el cementerio/hospital como espacio entre realidad y sueño}

La trama de la película sigue los pasos de un ama de casa solitaria de mediana edad, Jenjira (interpretada por la actriz no profesional Jenjira Pongpas), que se presenta voluntaria para atender a un grupo de soldados en un hospital rural que están aquejados de una misteriosa enfermedad del sueño que hace que se pasen dormidos casi todo el día. El hospital se ubica en una antigua escuela, construida a su vez sobre un antiguo cementerio de reyes locales. La película se centra en los cuidados que Jenjira dispensa a uno de los soldados, Itt, que no tiene familiares que lo atiendan, y con el que establece una extraña relación en los breves ratos en que despierta de ese estado que aunque ficcional parece asemejarse a una forma patológica extrema de narcolepsia (Foto 3).

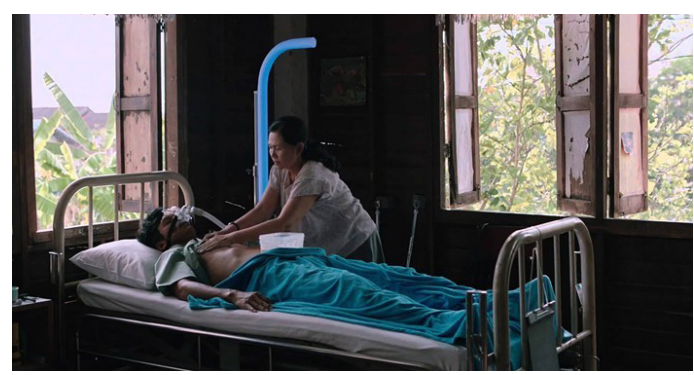

Foto 3. La protagonista, Jenjira Pongpas, asistiendo y cuidando al soldado dormido Itt
En la película, bajo el aspecto médico y hospitalario, aparentemente convencional, con médicos y enfermeras en batas blancas y respiradores y otros medios médicos, se ensayan extrañas terapias basadas en proyección de luces de neón fluorescentes afín de inducir "buenos sueños» en los soldados (Foto 4). A la vez hay una mujer espiritista capaz de comunicar entre los soldados dormidos y sus familiares, y que a la vez es capaz de «ver» el palacio en el que vivían los reyes y sobre el que se levanta el hospital. Esta mujer transmitirá, avanzada la película, su particular don a Jenjira, que será capaz entonces de "ver» también los sueños de su paciente ltt.

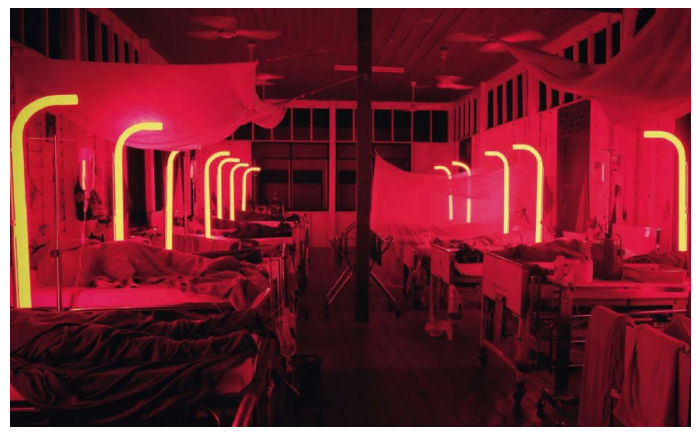

Foto 4. Terapia basada en proyección de luces fluorescentes en el hospital

Esta película incluye como Syndromes and a Century escenas muy realistas de consultas médicas en hospital, pocas veces tratadas en el cine con tanta naturalidad. Es un hospital rural similar al de la primera parte de dicha película, pero a menudo se insertan terapias alternativas inspiradas en meditación budista y magia animista.

En una escena, un hombre está dando una charla sobre meditación en la sala de los soldados dormidos a enfermeras y familiares de los enfermos. Se percibe aquí la influencia de la meditación budista de la escuela Theravada basadas en las técnicas introspectivas del vipassana incluyendo técnicas de visualizaciones. El hombre les dice: "Concéntrense en la parte de arriba de su cuerpo y en su cerebro, bajen los pensamientos hacia la

Rev. Med. Cine. 2021; 17 (2), 123-132 Ediciones Universidad de Salamanca / అ®@ J. Med. Mov., 2021; 17 (2), 123-132 [ 130 ] 
BAJO LA INFLUENCIA DEL BUDISMO Y EL ANIMISMO: CUERPO, ENFERMEDAD, MEDICINA Y CUIDADOS EN EL CINE DE APICHATPONG WEERASETHAKUL

ÁLVARO MALAINA

base de su cuerpo, a los pies. Ahora llévenlos a la cara... luego saquen la energía del cuerpo, llévenlo hasta las estrellas y luego vuelvan al lugar del cuerpo que les duele para curarlo, dejen que la energía los inunde, lo calme hasta que se sientan mejor. Una vez que se sientan mejor, muevan esa energía a la nuca. Que se concentre en ese lugar».

En otra escena, que muestra la influencia animista, vemos cómo Jenjira es visitada por dos diosas laosianas de un santuario cerca de un lago, para agradecerle las ofrendas de estatuillas de animales que les ha hecho a las dos estatuas que las representan en el santuario. Las diosas le dicen que los soldados no se curarán nunca de su enfermedad. Le explican que en el hospital antiguamente había un palacio y que estalló una guerra entre reinos que duró miles de años. Le explican que allí también se ubicaba un cementerio de reyes y que los espíritus de los reyes muertos inhalan energía de los soldados dormidos para librar sus batallas eternas.

Los soldados duermen mejor y tienen menos pesadillas, esa es la cura de su enfermedad, la terapia que buscan aplicar en el hospital por medio de la máquina y las lámparas de colores que les inducen buenos sueños. "O a lo mejor la medicina está funcionando» comenta una doctora mostrando así como en la película la medicina ortodoxa es puesta al mismo lugar que terapias alternativas, y siempre sin que una sustituya o rechace categóricamente a la otra, en un mismo plano horizontal no jerárquico.

\section{Conclusión}

Ong y Collier dicen que la era actual lo que produce es "ensamblajes globales» ${ }^{7}$ que combinan formas y normas racionales globales (sobre todo económicas y tecnológicas, pero también podrían ser científicas o médicas en nuestro caso) con entornos éticos, políticos y culturales situados localmente, formando híbridos culturales ${ }^{11}$, es decir sin generar homogeneización u occidentalización del mundo ni tampoco choques de civilizaciones y repliegues localistas y tradicionalistas.

Tenemos un ejemplo de ello en la medicina y una visualización de ello en el cine que se realiza en países no occidentales, como es el caso de Weerasethakul en Tailandia. Inspirándonos en la filosofía de Gilles Deleuze podemos describir su obra como «rizomas etno-cinematográficos», al estudiar, de forma muy realista, grupos humanos culturales con material cinematográfico, cruzando estilo hiperrealista casi documental con elementos oníricos, surrealistas, místicos y fantásticos, en un mismo plano horizontal sin jerarquización alguna.

La obra fílmica de Apichatpong pone la enfermedad y la terapia en el centro. En Syndromes and a Century recoge parte de las memorias de su infancia en la región de Isan, donde se combinaban prácticas biomédicas, budistas y animistas sin ninguna tensión ni contradicción. En Cemetery of Splendour profundiza, aún más, en su recorrido por la medicina, y crea incluso una enfermedad inexistente para su película como metáfora en gran parte política de la deriva autoritaria y militarista de su país, que habría sumido a gran parte de la sociedad en una especie de letargo narcótico ${ }^{12}$. Enfermedad que es tratada con medicina ortodoxa, pero también con técnicas de meditación y magia. Es de extraordinario interés en la era global en que vivimos, los acercamientos que el cine de Apichatpong hace a la medicina, mostrando sus expresiones complejas en realidades culturales no occidentales pero bajo la influencia determinante del modelo biomédico occidental.

\section{Referencias}

1. Teh D. Itinerant Cinema: The Social Surrealism of Apichatpong Weerasethakul. Third Text. 2011;25(5):595609.

2. Furhmann A. Gosthly Desires: Queer Sexuality and Vernacular Buddhism in Contemporary Thai Cinema. Durham: Duke University Press; 2016.

3. Ingawanij MA. Animism and the Performative Realist Cinema of Apichatpong Weerasethakul. En: Pick

Rev. Med. Cine. 2021; 17 (2), 123-132 Ediciones Universidad de Salamanca / ॠ@ J. Med. Mov., 2021; 17 (2), $123-132$ 


\section{BAJO LA INFLUENCIA DEL BUDISMO Y EL ANIMISMO: CUERPO, ENFERMEDAD, MEDICINA Y CUIDADOS EN EL CINE DE APICHATPONG WEERASETHAKUL \\ ÁLVARO MALAINA}

A, Narraway G, editores. Screening Nature: Cinema beyond the Human. Oxford: Berghahn Books; 2013. p. 91-109.

4. Said EW. Orientalismo. Barcelona: DeBolsillo; 2007.

5. Malaina A. Rizomas etno-cinematográficos: un estudio de Manta Ray (Puttiphong Aroonpheng, 2018) y Una tierra imaginada (Yeo Siew Hua, 2018). Área Abierta. Revista de Comunicación Audiovisual y Publicitaria. 2020;20(2):209-25.

6. Deleuze G, Guattari F. Mil Mesetas. Capitalismo y esquizofrenia. Valencia: Pre-textos; 1988.

7. Collier S, Ong A. Global Assemblages. Malden: Blackwell; 2005.
8. Laumakis SJ. An Introduction to Buddhist Philosophy. Cambridge: Cambridge University Press; 2008.

9. Bucknell R, Kang C. The Meditative Way: Readings in the Theory and Practice of Buddhist Meditation. Londres: Routledge; 2013.

10. Arhem K, Sprenger G. Animism in Southeast Asia. Londres: Routledge; 2015.

11. Pieterse JN. Globalization and Culture: Global Mélange. Lanham: Rowman \& Littlefield; 2003.

12. Mackris J. The Intersection of Politics and Trauma in the Films of Apichatpong Weerasethakul. Film Matters. 2017;8(1):35-8.

\begin{tabular}{|l|l|}
\hline & $\begin{array}{l}\text { Alvaro Malaina es doctor en Sociología por la Escuela de Altos Estudios en } \\
\text { Ciencias Sociales (EHESS) de París y por la Universidad Complutense de } \\
\text { Madrid. Ha sido investigador postdoctoral en el Departamento de Antro- } \\
\text { pología de la Universidad de California-Berkeley y actualmente es profesor } \\
\text { asociado de Sociología en la Universidad Complutense de Madrid, ejerciendo } \\
\text { docencia en el grado de Terapia Ocupacional de la Facultad de Medicina. Es } \\
\text { autor del libro Le paradigme de la complexité et la sociologie (L'Harmattan, } \\
\text { París, 2012), así como de publicaciones en diferentes revistas y libros. Sus } \\
\text { temas de investigación son la teoría sociológica y los cambios sociales en } \\
\text { la era de la globalización. }\end{array}$ \\
\hline
\end{tabular}

Rev. Med. Cine. 2021; 17 (2), 123-132 Ediciones Universidad de Salamanca / ๑®@ J. Med. Mov., 2021; 17 (2), 123-132 